\title{
Local Recovery of Sub-Crustal Stress Due to Mantle Convection from Satellite-to-Satellite Tracking Data
}

\author{
Michal ŠPRLÁK ${ }^{1}$ and Mehdi ESHAGH ${ }^{2}$ \\ ${ }^{1}$ NTIS - New Technologies for the Information Society, \\ Faculty of Applied Sciences, University of West Bohemia, Plzeň, Czech Republic \\ e-mail: sprlak@ntis.zcu.cz (corresponding author) \\ ${ }^{2}$ Department of Engineering Science, University West, Trollhättan, Sweden \\ e-mail: mehdi.eshagh@hv.se
}

\begin{abstract}
Two integral transformations between the stress function, differentiation of which gives the meridian and prime vertical components of the sub-crustal stress due to mantle convection, and the satellite-to-satellite tracking (SST) data are presented in this article. In the first one, the SST data are the disturbing potential differences between twin-satellites and in the second one the line-of-sight (LOS) gravity disturbances. It is shown that the corresponding integral kernels are well-behaving and therefore suitable for inversion and recovery of the stress function from the SST data. Recovery of the stress function and the stress components is also tested in numerical experiments using simulated SST data. Numerical studies over the Himalayas show that inverting the disturbing potential differences leads to a smoother stress function than from inverting LOS gravity disturbances. Application of the presented integral formulae allows for recovery of the stress from the satellite mission GRACE and its planned successor.
\end{abstract}

Key words: integral equation, upward/downward continuation, integral kernel, truncation error, GRACE satellite mission, stress function. 


\section{INTRODUCTION}

The sub-crustal stress due to mantle convection is one of the important geophysical phenomenae. It allows for interpretation of seismicity, volcanicity, kimberlite magmatism, ore formation, tectonic and magnetic features (Liu 1977). Runcorn $(1964,1967)$ was one of the pioneers who proposed modelling the sub-crustal shear stress components in terms of spherical harmonic coefficients of gravity field. His formula was successfully applied, e.g., by Liu et al. (1976), Liu (1977, 1978, 1980a, b), and Huang and Fu (1982). Others have also worked on the relation between the stress and other types of gravity data. McKenzie (1967) mentioned that the long wavelength harmonics of the external gravity field cannot be supported by the strength of the lithosphere. Review of stress estimates based on gravity observations and isostatic compensation based on surface deformation was provided by McNutt (1980). Souriau and Souriau (1983) studied correlations between surface tectonics and geoid undulations in terms of harmonic expansion. In a similar way, Ricard et al. (1984) investigated the connection between the lithospheric stress and geoidal undulation in terms of spherical harmonics. Mathematical model in terms of a harmonic expansion for convection field of the mantle was provided by $\mathrm{Fu}(1986,1990)$.

Pick and Charvátová-Jakubcová (1988) and Pick (1994) provided integral transformations between geoid or gravity anomaly and the stress components to reduce the contribution of the far-zone gravitational effects. Eshagh (2014) related gradiometric data, such as those provided by the Gravity field and steady-state Ocean Circulation Explorer (GOCE; ESA 1999) satellite mission, and the stress components by integral transformations. In fact, he proposed a scalar function, called stress function (SF), differentiating of which stress components may be obtained. Eshagh (2015) developed this theory further to use the existing model of Moho discontinuity for sub-crustal stress determination. Eshagh and Tenzer (2015) used this method for determination of the sub-crustal stress in different regions with proper geological interpretations. Tenzer and Eshagh (2015) investigated the subduction generated by sub-crustal stress under Taiwan. Eshagh (2016) presented some integral formulae for recovering the sub-crustal stress using terrestrial gravimetric data and applied them for stress modelling under Iran. Tenzer et al. (2015) applied this method for evaluation of stress fields on Mars. Eshagh and Romeshkani (2015) used the real GOCE data for recovering the sub-lithospheric stress field in Iran.

In this article, we discuss a model for recovery of the sub-crustal stress from satellite-to-satellite tracking (SST) data, e.g., such as those available from the GRAvity field and Climate Experiment (GRACE; Tapley et al. 2004). From the mathematical point of view, we provide two integral trans- 
formations of the SF onto the SST data, which are in the form of gravitational potential differences and the line-of-sight (LOS) gravitational accelerations. Local recovery of the sub-crustal stress and/or any other type of gravity data is beneficial as: (a) global coverage of the data is not required; (b) the data with good coverage, uniform distribution and accuracies is used; and (c) local features are more visible. The third reason is highly dependent on the satellite altitude and type of the satellite data. The satellite data may represent the deep interior structure of the Earth much better than the local terrestrial data and especially the GRACE data are more sensitive to the long wavelength structure of the stress field occurring beneath the crust.

We extend the geophysical applications of the GRACE mission, such as those improving models of crustal thickness (Shin et al. 2007, Block et al. 2009, Tedla et al. 2011, Arabelos and Tsoulis 2013, Zhao 2013), lithospheric structures (Ricard et al. 2006, Kiamehr et al. 2008, Braitenberg and Ebbing 2009a, b; von Frese 2009, Kaban et al. 2010, Kaban and Trubitsyn 2012, Köther et al. 2012, Tondi et al. 2012), and our understanding of tectonic (Mikhailov et al. 2004, Kiamehr and Sjöberg 2006, Choi et al. 2006, McAdoo et al. 2008) and seismic processes (see, e.g., Sun and Okubo 2004, Chen et al. 2007, Han et al. 2008, Panet et al. 2010, Matsuo and Heki 2011, Wang et al. 2012, Mikhailov et al. 2013, Dai et al. 2014, Li et al. 2014, Sun 2014).

In Section 2, we describe the SST data and present its relations to the disturbing gravitational field quantities such as disturbing potential differences (DPD) and LOS gravity disturbances. The new integral formulae are developed in Section 3 relating these quantities to the SF (Eshagh and Tenzer 2015). Here, we also study the behaviour of the corresponding subintegral kernels in the spatial domain. The derived integral formulae are numerically tested in closed-loop simulations in Section 4. We investigate the accuracy of our algorithms by calculating the SST data assuming the SF is known, i.e., the direct problem. We also consider the estimation of the SF and the stress components from known SST data, i.e., the inverse problem. Contributions of this article are summarised in Section 5. Application of the mathematical apparatus to real SST data and geophysical interpretation of the recovered stress function is out of the scope of this paper.

\section{SATELLITE-TO-SATELLITE TRACKING}

SST is an inter-satellite measurement technique for precise determination of the satellite coordinates. It can be performed in two modes: (i) the high-low mode, when position of a low orbiting satellite is determined by satellites at higher altitudes; and (ii) the low-low mode, when two low-orbiting satellites follow each other in approximately the same orbit (see Reigber 1989, Seeber 2003). SST in high-low mode has been successfully realised by the satellite 
mission CHAMP (Challenging Minisatellite Payload; Reigber et al. 2002). SST in low-low and high-low modes have been exploited on-board of GRACE mission mapping the Earth's gravitational field and GRAIL (Gravity Recovery and Interior Laboratory; Zuber et al. 2013) mission, which focused on the Moon's gravitational field.

The high-low SST provides position, velocity and acceleration vectors in the geocentric Earth-fixed frame, namely:

$$
\mathbf{x}=[x, y, z]^{T}, \dot{\mathbf{x}}=[\dot{x}, \dot{y}, \dot{z}]^{T}, \ddot{\mathbf{x}}=[\ddot{x}, \ddot{y}, \ddot{z}]^{T} .
$$

These are derived from Global Navigation Satellite Systems (GNSS) carrier phase observations. The inter-satellite ranging system between a pair of low-orbiting satellites provides the time series of the following quantities, i.e., the low-low SST data:

$$
\rho=|\delta \mathbf{x}|, \quad \dot{\rho}=\delta \dot{\mathbf{x}} \cdot \mathbf{e}, \quad \ddot{\rho}=\delta \ddot{\mathbf{x}} \cdot \mathbf{e}+\frac{|\delta \dot{\mathbf{x}}|^{2}-\dot{\rho}^{2}}{\rho} .
$$

Here, $\rho$ is the inter-satellite range, $\dot{\rho}$ and $\ddot{\rho}$ represent its first and second time derivatives, i.e., the inter-satellite velocity difference and inter-satellite acceleration difference (being derived from the inter-satellite range), e stands for the LOS inter-satellite unit vector and

$$
\delta \mathbf{x}=\mathbf{x}_{2}-\mathbf{x}_{1}, \quad \delta \dot{\mathbf{x}}=\dot{\mathbf{x}}_{2}-\dot{\mathbf{x}}_{1}, \quad \delta \ddot{\mathbf{x}}=\ddot{\mathbf{x}}_{2}-\ddot{\mathbf{x}}_{1},
$$

where the subscript 1 designates the leading satellite and the subscript 2 is assigned to the trailing satellite.

For gravity field modelling, the SST data should be mathematically related to some quantities of the field. The range-rates and velocity measurements have the simplest mathematical relations to the differences of the gravitational potential $V$ between the pair of satellites (see, e.g., Wolff 1969, Fischell and Pisacane 1978, Rummel 1980):

$$
\delta V(\mathbf{x})=\left|\dot{\mathbf{x}}_{1}\right| \dot{\rho} .
$$

Note that Eq. 4 holds only approximately. A rigorous model for $\delta V$ was provided and extensively analysed by Jekeli (1999). This model should be preferred for gravity field modelling purposes. Alternatively, the low-low and high-low SST data may rigorously be combined to provide differences of gravitational gradients projected onto the LOS inter-satellite unit vector (see, e.g., Hajela 1974, Rummel 1980, Keller and Sharifi 2005):

$$
\frac{|\delta \dot{\mathbf{x}}|-\dot{\rho}^{2}-\rho \ddot{\rho}}{\rho}=\frac{\delta \mathbf{x} \cdot \delta \nabla V(\mathbf{x})}{\rho}=\mathbf{e} \cdot \delta \nabla V(\mathbf{x}) .
$$


The right hand-side of Eq. 5 is also referred to as LOS gravitational acceleration.

In the following, we will derive the integral formulae between the SF, whose derivatives correspond to the components of the stress due to mantle convection, and the SST data in the form of Eqs. 4 and 5. We introduce the DPD by $\delta T$ and LOS gravity disturbances by $\mathbf{e} \cdot \delta \nabla T$, where $T$ stands for the disturbing gravitational potential. Both, the DPD and LOS gravity disturbances, can be obtained by subtracting the normal gravitational potential, e.g., generated by the GRS80 reference ellipsoid (Moritz 2000), from both sides of Eqs. 4 and 5.

\section{MATHEMATICAL DEVELOPMENTS}

Here, we develop the mathematical relations between each one of $\delta T$ and e $\delta \nabla T$ and the SF, which are in fact of integral types. Also, we present the behaviour of the integral kernels to have an idea about the far-zone contribution of the integral formulae.

\subsection{Integral transformation of the stress function onto disturbing gravitational potential}

In this section, we focus on deriving the mathematical link between the SF and the disturbing gravitational potential. The spherical harmonic expansion of the disturbing gravitational potential is:

$$
T(\mathbf{x})=\sum_{n=2}^{\infty} \sum_{m=-n}^{n}\left(\frac{R}{r}\right)^{n+1} \bar{T}_{n m} \bar{Y}_{n m}(\Omega),
$$

where $R$ is the radius of the reference sphere, the triplet $r, \Omega=(\varphi, \lambda)$ represents the spherical coordinates, i.e., the geocentric radius, spherical latitude $\varphi$ and longitude $\lambda$, and they are presented by the vector $\mathbf{x}=\mathbf{x}(r, \Omega)$ on the lefthand side of Eq. 6. $\bar{T}_{n m}$ stand for the fully-normalised spherical harmonic coefficients of the disturbing gravitational potential of degree $n$ and order $m$. These are related to the conventional dimensionless fully-normalised spherical harmonic coefficients $\bar{C}_{n m}$ by the following transformation: $\bar{T}_{n m}=\frac{G M}{R} \bar{C}_{n m}$, where $G$ stands for the Newtonian gravitational constant and $M$ is the mass of the Earth including oceans and atmosphere. The symbol $\bar{Y}_{n m}$ stands for the fully-normalised surface spherical harmonics. The summation in Eq. 6 starts from degree 2, meaning that the mass of the Earth equals to the mass of the reference ellipsoid and the frame in which the coordinates are defined is geocentric. 
Similarly, the SF may be expanded into the following spherical harmonic series (Eshagh 2014):

$$
S(\mathbf{x}, D)=\frac{R \kappa}{G M} \sum_{n=2}^{\infty} \sum_{m=-n}^{n} v_{n} \bar{T}_{n m} \bar{Y}_{n m}(\Omega)=\sum_{n=2}^{\infty} \sum_{m=-n}^{n} \bar{S}_{n m} \bar{Y}_{n m}(\Omega),
$$

where

$$
\kappa=\frac{M g}{4 \pi[R-D]^{2}}, \quad v_{n}=\frac{2 n+1}{n+1}\left[\frac{R}{R-D}\right]^{n+1},
$$

with $g$ being the mean value of gravity, $D$ is the normal or mean Moho depth and $\bar{S}_{n m}$ are the fully-normalised harmonic coefficients of the SF. By comparing Eqs. 6 and 7 it will not be difficult to find that:

$$
\bar{T}_{n m}=\frac{G M}{R \kappa v_{n}} \bar{S}_{n m} .
$$

Equation 9 shows the direct relation between the spherical harmonic coefficients of the SF and those of the disturbing gravitational potential. However, we can find an integral formula by transforming the SF onto the disturbing gravitational potential in the spatial domain. First, Eq. 9 is inserted back into Eq. 6, and by considering that:

$$
\bar{S}_{n m}=\frac{1}{4 \pi} \int_{\Omega^{\prime}} S\left(\mathbf{x}^{\prime}, D\right) \bar{Y}_{n m}\left(\Omega^{\prime}\right) \mathrm{d} \Omega\left(\mathbf{x}^{\prime}\right),
$$

where $\mathbf{x}^{\prime}=\mathbf{x}\left(R, \Omega^{\prime}\right)$ assigns the position of an integration element on the surface of the reference sphere $\Omega^{\prime}$, we have:

$$
T(\mathbf{x})=\frac{G M}{4 \pi R \kappa} \sum_{n=2}^{\infty} \frac{1}{v_{n}}\left(\frac{R}{r}\right)^{n+1} \int_{\Omega^{\prime}} S\left(\mathbf{x}^{\prime}, D\right) \sum_{m=-n}^{n} \bar{Y}_{n m}\left(\Omega^{\prime}\right) \bar{Y}_{n m}(\Omega) \mathrm{d} \Omega\left(\mathbf{x}^{\prime}\right) .
$$

Now, according to the addition theorem of the surface spherical harmonics (Hofmann-Wellenhof and Moritz 2005, p. 26):

$$
P_{n, 0}(\cos \psi)=\frac{1}{2 n+1} \sum_{m=-n}^{n} \bar{Y}_{n m}(\Omega) \bar{Y}_{n m}\left(\Omega^{\prime}\right),
$$

where $P_{n, 0}$ are the associated Legendre functions of the first kind of degree $n$ and order 0 (also known as Legendre polynomials) and $\psi$ is the spherical distance between the geocentric directions $\Omega$ and $\Omega^{\prime}$, we get:

$$
T(\mathbf{x})=\frac{G M}{4 \pi R \kappa} \int_{\Omega^{\prime}} S\left(\mathbf{x}^{\prime}, D\right) K(t, u) \mathrm{d} \Omega\left(\mathbf{x}^{\prime}\right) .
$$


The series representation of the integral kernel $K(t, u)$ is of the form:

$$
K(t, u)=\sum_{n=2}^{\infty} t^{n+1}(n+1) P_{n, 0}(u),
$$

with variables $t$ and $u$ defined as follows:

$$
t=t\left(\mathbf{x}, \mathbf{x}^{\prime}\right)=\frac{\left|\mathbf{x}^{\prime}\right|}{|\mathbf{x}|}=\frac{R}{r}, \quad u=u\left(\mathbf{x}, \mathbf{x}^{\prime}\right)=\frac{\mathbf{x} \cdot \mathbf{x}^{\prime}}{|\mathbf{x}|\left|\mathbf{x}^{\prime}\right|}=\cos \psi .
$$

We can also find a closed-form formula for the kernel of Eq. 14. To do so, we use the following identities, see, e.g., (Pick et al. 1973):

$$
\begin{aligned}
& \sum_{n=2}^{\infty} t^{n+1} P_{n, 0}(u)=t\left(\frac{1}{g}-1-t u\right), \\
& \sum_{n=2}^{\infty} n t^{n+1} P_{n, 0}(u)=t^{2}\left(\frac{u-t}{g^{3}}-u\right),
\end{aligned}
$$

where

$$
g=g(t, u)=\sqrt{1-2 t u+t^{2}} .
$$

Exploiting Eqs. 16 and 17, the infinite series of Eq. 14 may be written in the closed form:

$$
K(t, u)=-t\left(1+2 t u-\frac{1-t u}{g^{3}}\right) .
$$

\subsection{Integral transformation of the stress function onto SST data}

By using the integral formula of Eq. 13 we can find corresponding integral transformations between the SF and the SST data in the form of either the DPD $(\delta T)$ or LOS gravity disturbance $(\mathbf{e} \cdot \delta \nabla T)$. In the following we will develop these transformations.

\subsubsection{Integral transformation of the stress function onto disturbing potential differences}

Considering two low-orbiting satellites, $\delta T$ will be nothing else than a difference between disturbing gravitational potentials at the satellites' locations based on Eq. 13. In such a situation $\delta T$ is:

$$
\delta T(\mathbf{x})=\frac{G M}{4 \pi R \kappa} \int_{\Omega^{\prime}} S\left(\mathbf{x}^{\prime}, D\right) \delta K(t, u) \mathrm{d} \Omega\left(\mathbf{x}^{\prime}\right) .
$$


It is seen that $\delta T$ leads to an integral kernel difference $\delta K(t, u)$, which is a function of three points, i.e., the integration element $\mathbf{x}^{\prime}$ and the position vectors $\mathbf{x}_{1}$ and $\mathbf{x}_{2}$ of both satellites; see also Eq. 15 for the definition of variables $t$ and $u$.

To better understand the properties of the integral formula of Eq. 20 we have investigated the behaviour of the integral kernel $\delta K(t, u)$. For this purpose, we have selected one particular formation of GRACE twin satellites from the product GNV1B (GPS Navigation Data Format Record; Case et al. $2010)$ at GPS time $t_{\mathrm{GPS}}=316363415 \mathrm{~s}$. The spherical coordinates of the leading satellite are $r_{1}=6854183.3 \mathrm{~m}, \varphi_{1} \approx 0.30^{\circ}, \lambda_{1} \approx 5.05^{\circ}$, while the spherical coordinates of the trailing satellite are $r_{2}=6855126.8 \mathrm{~m}, \varphi_{2} \approx-1.79^{\circ}$, $\lambda_{2} \approx 5.10^{\circ}$. It follows that the barycentre of the satellite pair, i.e., the arithme-
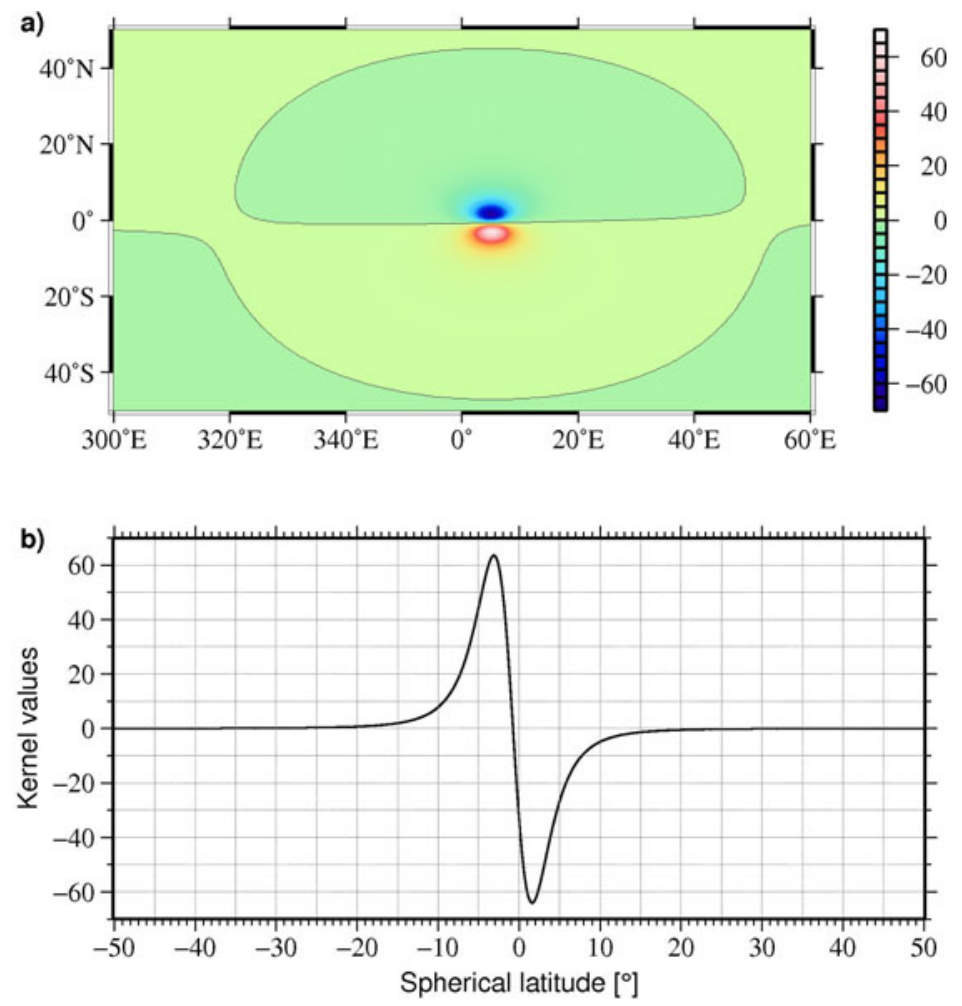

Fig. 1. Graphical illustration of the integral kernel $\delta K$ based on Eq. 20: (a) the total value of the kernel, (b) profile along the meridian with constant longitude $\lambda=5.125^{\circ}$. The spherical coordinates of the leading satellite are $r_{1}=6854183.3 \mathrm{~m}$, $\varphi_{1} \approx 0.30^{\circ}, \lambda_{1} \approx 5.05^{\circ}$. The spherical coordinates of the trailing satellite are $r_{2}=6855126.8 \mathrm{~m}, \varphi_{2} \approx-1.79^{\circ}, \quad \lambda_{2} \approx 5.10^{\circ}$. Value of the mean Moho depth is $D=30000 \mathrm{~m}$ and the radius of the reference sphere is $R=6378136.3 \mathrm{~m}$. The black solid line in Fig. 1a indicates the zero crossings of the integral kernel. 
tic average of angular spherical coordinates of both satellites, is defined by the position $\varphi_{\mathrm{B}} \approx-0.75^{\circ}, \lambda_{\mathrm{B}} \approx 5.08^{\circ}$. Next, we have assumed that the value of the mean Moho depth is $D=30000 \mathrm{~m}$ and the radius of the reference sphere is $R=6378136.3 \mathrm{~m}$. Integral kernel $\delta K(t, u)$ in the closed-form based on Eq. 19 has been computed globally with step $0.25^{\circ}$ in both angular directions and depicted in the area bounded by spherical coordinates $\varphi \in$ $\left[-50.125^{\circ}, 50.125^{\circ}\right], \lambda \in\left[-60.125^{\circ}, 60.125^{\circ}\right]$; see Fig. 1 . Despite of the fact that the kernel $K(t, u)$ is isotropic, the difference $\delta K(t, u)$ is not isotropic anymore and leads to the azimuthal dependence illustrated in Fig. 1a. The extreme behaviour of the integral kernel is seen in the along-track direction that almost coincides with latitude direction. Obviously, value of the integral kernel is zero at the barycentre of the two satellites' positions. Moving from the barycentre along-track by approximately $2.5^{\circ}$, the total integral kernel reaches its extreme values. With increasing distance from the barycentre, one may observe the decay to zero. These facts are clearly demonstrated in Fig. 1b, which represents the behaviour of the integral kernel along the meridian with constant longitude $\lambda=5.125^{\circ}$. The integral kernel is much smoother with significantly reduced magnitudes and values close to zero in the cross-track (longitude) direction. Implications of the integral kernel behaviour for the recovery of the SF are further discussed in Section 4.2.

\subsubsection{Integral transformation of the stress function onto LOS gravity disturbances}

To obtain the integral formula connecting the SF to the LOS gravity disturbance we use the following operator (Šprlák and Novák 2014a, b):

$$
\mathbf{e} \cdot \delta \nabla=\frac{1}{R} \delta\left(e_{x} t \sqrt{1-u^{2}} \cos \alpha \frac{\partial}{\partial u}+e_{y} t \sqrt{1-u^{2}} \sin \alpha \frac{\partial}{\partial u}-e_{z} t^{2} \frac{\partial}{\partial t}\right) .
$$

In the last equation, $e_{x}, e_{y}$, and $e_{z}$ are the components of the LOS intersatellite unit vector in the spherical local north-oriented reference frame, i.e., the frame with origin at satellite's center of mass, with $x$ axis directed north, $y$ axis pointing west, and $z$ axis pointing in the radial direction, $\alpha=\alpha\left(\Omega, \Omega^{\prime}\right)$ is the direct azimuth, and $t, u$ are the substitutions defined by Eq. 15. Applying the differential operator of Eq. 21 onto Eq. 13 we get:

$$
\text { e. } \delta \nabla T(\mathbf{x})=\frac{G M}{4 \pi R^{2} \kappa} \int_{\Omega^{\prime}} S\left(\mathbf{x}^{\prime}, D\right) \delta\left\{K^{u}(t, u)\left[e_{x} \cos \alpha+e_{y} \sin \alpha\right]-e_{z} K^{t}(t, u)\right\} \mathrm{d} \Omega\left(\mathbf{x}^{\prime}\right) \text {. }
$$

The last equation relates the SF to the LOS gravity disturbance. Obviously, the integral kernel is azimuth-dependent due to the presence of the trigonometric functions of the direct azimuth $\alpha$. Similar to Eq. 20, the integral 
kernel is a function of three points, i.e., the integration element $\mathbf{x}^{\prime}$ and the position vectors $\mathbf{x}_{1}$ and $\mathbf{x}_{2}$ of both satellites. In addition, the integral kernel is composed of two isotropic kernels, which are defined as follows:

$$
\begin{aligned}
& K^{u}(t, u)=t \sqrt{1-u^{2}} \frac{\partial K(t, u)}{\partial u}=\sum_{n=2}^{\infty} t^{n+2}(n+1) P_{n, 1}(u)=-t^{3} \sqrt{1-u^{2}}\left(2-\frac{2-t u-t^{2}}{g^{5}}\right), \\
& K^{t}(t, u)=t^{2} \frac{\partial K(t, u)}{\partial t}=\sum_{n=2}^{\infty} t^{n+2}(n+1)^{2} P_{n, 0}(u)=-t^{2}\left\{1+4 t u-\frac{1}{g}-\frac{t}{g^{3}}\left[3 u-4 t+\frac{3 t(t-u)^{2}}{g^{2}}\right]\right\} .
\end{aligned}
$$

Equations 23 and 24 define the spectral and closed forms of the isotropic kernels $K^{u}(t, u)$ and $K^{t}(t, u)$. The spectral forms immediately follow by applying the first equalities in Eqs. 23 and 24 to Eq. 14. On the other hand, the closed expressions may be derived making use of the identities of Eqs. 16 and 17 together with (see, e.g., Pick et al. 1973, Šprlák and Novák 2014b):

$$
\begin{gathered}
\sum_{n=2}^{\infty} n^{2} t^{n+1} P_{n, 0}(u)=-t^{2}\left[u-\frac{u-2 t}{g^{3}}-\frac{3 t(t-u)^{2}}{g^{5}}\right], \\
\sum_{n=2}^{\infty} t^{n+1} P_{n, 1}(u)=-t^{2} \sqrt{1-u^{2}}\left(1-\frac{1}{g^{3}}\right), \\
\sum_{n=2}^{\infty} n t^{n+1} P_{n, 1}(u)=-t^{2} \sqrt{1-u^{2}}\left(1-\frac{1}{g^{3}}-\frac{3 t(u-t)}{g^{5}}\right) .
\end{gathered}
$$

We now demonstrate properties of the integral formula of Eq. 22 by investigating the behaviour of its integral kernel. The integral kernel has been evaluated globally with equiangular step of $0.25^{\circ}$ and illustrated in the spatial domain bounded by spherical coordinates $\varphi \in\left[-30.125^{\circ}, 30.125^{\circ}\right]$, $\lambda \in\left[-30.125^{\circ},-40.125^{\circ}\right]$. Note that the other kernel parameters, i.e., positions of the satellite pair, the mean Moho depth $D$ and the radius of the reference sphere $R$, have the same values as those already described for the integral kernel $\delta K(t, u)$; see Section 3.2.1.

The total integral kernel of Eq. 22 is illustrated in Fig. 2a, which shows its azimuth-dependent behaviour. The most significant values are again visible in the along-track direction. This direction is approximated by a meridian of constant longitude $\lambda=5.125^{\circ}$ in Fig. $2 \mathrm{~b}$. We may observe that the integral kernel of Eq. 22 reaches its negative extreme value at the barycentre and then reaches local positive extremes approximately $4.5^{\circ}$ from the barycentre. The kernel then decreases to zero with increasing distance from the barycen- 
a)
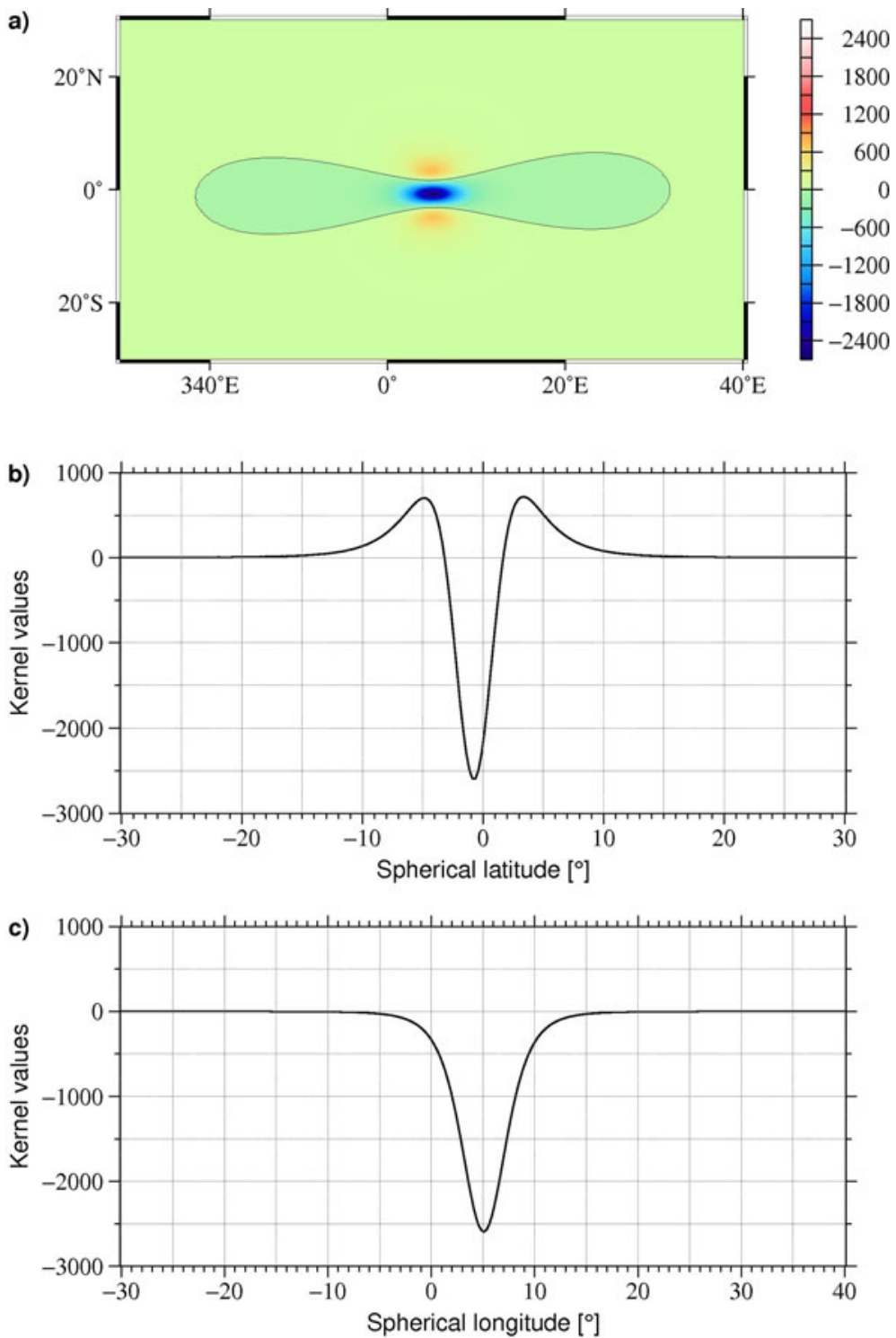

Fig. 2. Graphical illustration of the integral kernel of Eq. 22: (a) the total value of the kernel, (b) profile along the meridian with constant spherical longitude $\lambda=5.125^{\circ}$, (c) profile along the parallel with constant spherical latitude $\varphi=-0.625^{\circ}$. The spherical coordinates of the leading satellite are $r_{1}=6854183.3 \mathrm{~m}, \varphi_{1} \approx 0.30^{\circ}, \lambda_{1} \approx$ $5.05^{\circ}$. The spherical coordinates of the trailing satellite are $r_{2}=6855126.8 \mathrm{~m}, \varphi_{2} \approx$ $-1.79^{\circ}, \lambda_{2} \approx 5.10^{\circ}$. Value of the mean Moho depth is $D=30000 \mathrm{~m}$ and the radius of the reference sphere is $R=6378136.3 \mathrm{~m}$. The black solid line in Fig. 2a indicates the zero crossings of the integral kernel. 
tre. Figure 2c demonstrates the behaviour of the integral kernel along the parallel with constant latitude $\varphi=0.625^{\circ}$. This direction represents approximately the cross-track direction and at the same time it is very close to the barycentre. It is seen that in this direction the integral kernel decays to zero faster since the local positive extremes are completely reduced. Behaviour of the integral kernel and its effect on the recovery of the SF are discussed in Section 4.2.

\section{NUMERICAL EXPERIMENTS}

Here, we divide our numerical studies into two parts. First, we will test the direct problem in which the generated SF by EGM2008 (Pavlis et al. 2012) is used to reproduce the SST data. In the second numerical experiment, we try to recover the SF by inverting $\delta T$ and $\mathbf{e} \cdot \delta \nabla T$ based on the mathematical apparatus presented in the previous section.

\subsection{Testing the direct problem}

The presented theories have been practically tested in a numerical experiment. First, the correctness of our mathematical derivations has been checked as the direct problem, i.e., direct integration of the SF for calculation of the SST data based on Eqs. 20 and 22. The SF has been generated from EGM2008 global gravity field model (GGFM) up to degree and order $(\mathrm{d} / \mathrm{o}) 180$ in a global equiangular grid with spacing of $1^{\circ}$. For evaluation of the SF, we assumed that the value of the mean Moho depth is $D=30000 \mathrm{~m}$ and the radius of the reference sphere is $R=6378136.3 \mathrm{~m}$. Next, the true $\delta T$ and the true e $\delta \nabla T$ have been computed from EGM2008 up to d/o 180 at the locations given by real GRACE orbit available from the product GNV1B. Every fifth data record in the period from 1 January 2010 until 30 June 2010 have been considered and restricted to the area bounded by spherical coordinates $\varphi \in\left[5^{\circ}, 57^{\circ}\right]$ and $\lambda \in\left[55^{\circ}, 115^{\circ}\right]$. The restricted domain covers the area of India, Himalayas and Southeast Asia and contains 30036 points in total. The statistics of the SST data is presented in Table 1 and their spatial behaviour is depicted in Fig. 3. We note that the values of the SST data were interpolated from the original locations along the orbit into an equiangular grid with sampling of $0.25^{\circ}$ for better visualisation purposes. We also note that values of $\delta T$ have opposite sign for descending and ascending tracks. In Fig. 3a we have opted for ascending tracks when interpolating $\delta T$ and Fig. $3 \mathrm{~b}$ is a similar map but for $\mathbf{e} \cdot \delta \nabla T$. We observe that the map of $\delta T$, presented in Fig. 3a is smoother than that of $\mathbf{e} \cdot \delta \nabla T$ in Fig. $3 \mathrm{~b}$ as expected.

In a further step, we have performed the global integration of the SF based on integral formulae of Eqs. 20 and 22. For this purpose, numerical integration method has been implemented in a computer program. Subsequent- 
Statistics of the differences between the SST data obtained by numerical integration and their true values

\begin{tabular}{|c|c|c|c|c|c|c|}
\hline SST data & Equation & Units & Minimum & Maximum & Mean & Std. dev. \\
\hline \multirow{2}{*}{$\delta T$} & Signal & \multirow{2}{*}{$\mathrm{m}^{2} \mathrm{~s}^{-2}$} & -44.938 & 46.892 & 0.602 & 18.123 \\
\cline { 2 - 5 } & 20 & & -0.005 & 0.005 & 0.000 & 0.001 \\
\hline \multirow{2}{*}{ e. $\delta \nabla T$} & Signal & \multirow{2}{*}{$\mathrm{mGal}$} & -10.196 & 8.135 & 0.894 & 2.449 \\
\cline { 2 - 5 } & 22 & & -0.096 & 0.102 & 0.004 & 0.012 \\
\hline
\end{tabular}

Notes: Closed isotropic kernels were exploited in the numerical integration. The true values were synthesised from EGM2008 up to d/o 180. Statistics of the true values are given in the rows called "Signal".
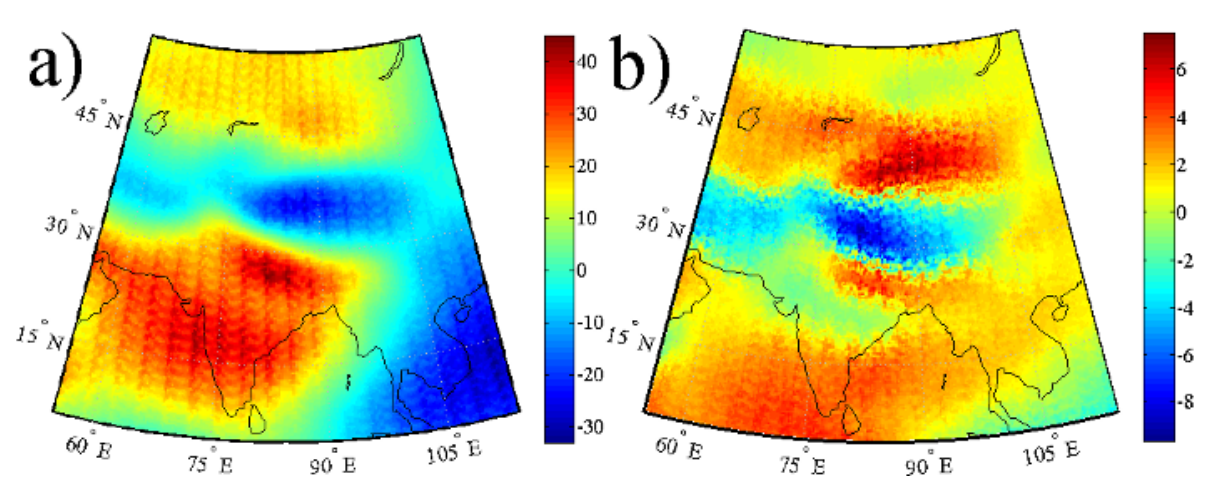

Fig. 3: (a) Disturbing potential differences $(\delta T)\left[\mathrm{m}^{2} \mathrm{~s}^{-2}\right]$; and (b) LOS gravity disturbance $(\mathbf{e} \cdot \delta \nabla T)$ [mGal].

ly, $\delta T$ and $\mathbf{e} \cdot \delta \nabla T$ from the global integration have been compared with the true values. Statistics of the differences is presented in Table 1. The standard deviation of differences reaches $0.001 \mathrm{~m}^{2} \mathrm{~s}^{-2}$ for $\delta T$ and $0.012 \mathrm{mGal}$ for e. $\delta \nabla T$. The relative accuracy, defined as the ratio of the standard deviation of differences to the standard deviation of the signal, is two orders higher for e $\delta \nabla T$. This indicates that the performance of the numerical integration for $\delta T$ is better. We suppose that the different performance is due to different spectral sensitivity of the quantities in Eqs. 20 and 22. Evaluation of $\delta T$ is more precise as the SF is of similar spectral content. On the other hand, e $\delta \nabla T$ is more sensitive to higher frequencies of the gravity field as compared to the SF. Evaluation of $\mathbf{e} \cdot \delta \nabla T$ may therefore be less accurate from a numerical point of view. 


\subsection{Solution of the inverse problem}

The integrals 20 and 22 should be inverted to recover the SF. To do so, they are discretised with an equiangular resolution of $1^{\circ}$ that corresponds to spherical harmonic expansion up to degree 180 . Recovering SF with higher resolution is not meaningful as the GRACE satellite data are not sensitive to higher degrees. The synthesised GRACE data on their real orbit in the area $\varphi \in\left[5^{\circ}, 57^{\circ}\right]$ and $\lambda \in\left[55^{\circ}, 115^{\circ}\right]$ are considered as the observations. The discretised integrals represent observation equations connecting the observations to the SF in the same area. According to the size of the study area and the resolution of SF, 3120 values should be determined for SF and the number of synthesised data is 30036 , which means that we have 26916 data more than the unknown values of SF. Therefore, the systems of equations, organised by the discretisation of the integral formulae, should be solved by the least-squares method. The determinant of the coefficients matrix of the system is very small which makes the inversion process numerically unstable with a condition number of $2 \times 10^{14}$. Regularisation is a method for solving such unstable systems. In this study, we use Tikhonov regularisation (Tikhonov 1963) for stabilising the system and L-curve (Hansen 1998) for estimating the regularisation parameter. For inverting our systems of equations we have used Regularisation Tools (Hansen 2007) of MATLAB. For more information about regularisation, see, e.g., Hansen (1998), Bouman (1998) or Eshagh (2011a).

It should be noted that we have simulated $\delta T$ and $\mathbf{e} \cdot \delta \nabla T$ using the EGM2008 model, while real GRACE orbits have been exploited as the purpose of this study is to investigate how successful our methods for recovering SF are for the noise-free scenario. In this way, we can judge better the theoretical apparatus derived in Section 3 rather than working with the real GRACE data which are of different nature and could make our primary investigations complicated. Recovery of SF from noisy SST data and application of the derived theoretical apparatus for real GRACE data is left for our future research.

\subsubsection{Spatial truncation error}

The spatial truncation error is a serious problem in inversion of the spherical integrals like those in Eqs. 20 and 22, in addition to the instability of system of equations organised from them. This is due to lacking data in the integration domain of integral formulae and occurs mainly in the marginal parts of the inversion area. The simplest way for reducing this error is to perform the inversion process in whole area and select the results located in the central part as the final result. Eshagh (2011b) showed that the truncation error may be reduced well when data and unknowns are located within the same area. 

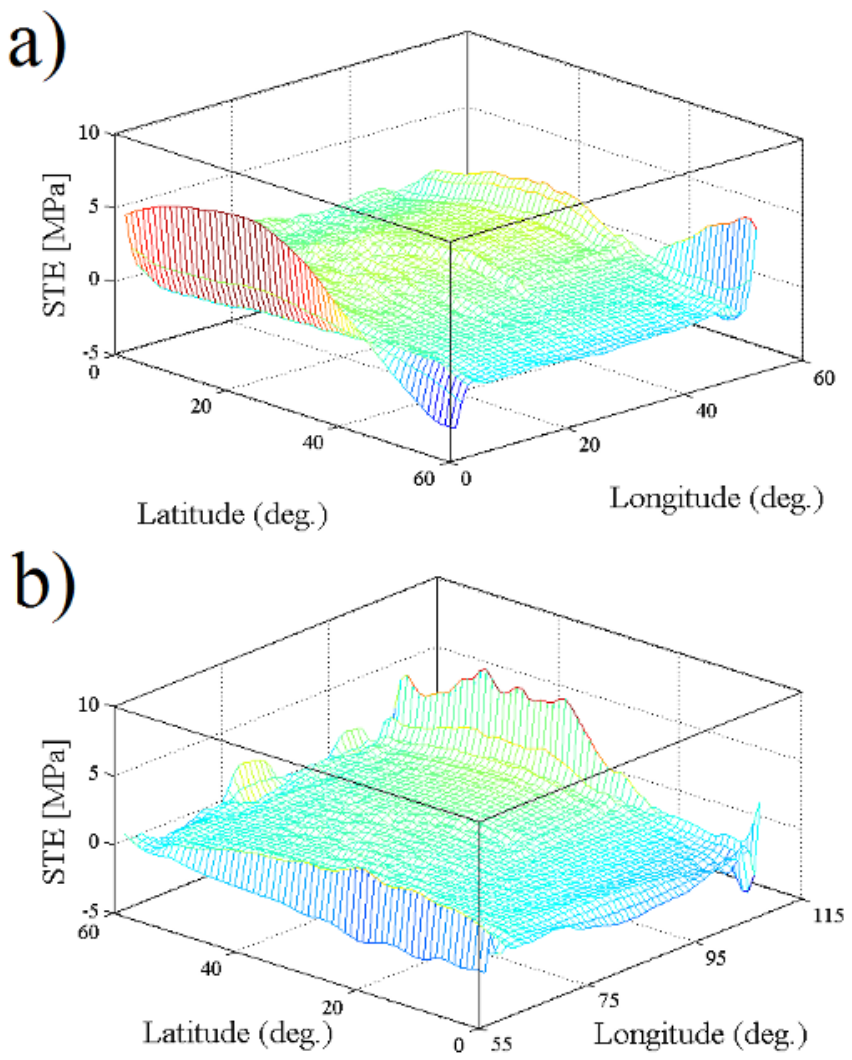

Fig. 4. Spatial truncation error (STE) of recovered SF by inverting integral of: (a) Eq. 20, and (b) Eq. 22.

Also, spatial behaviour of the corresponding integral kernel has a tremendous role to reduce the truncation error. Based on Eshagh (2011b), the truncation error may be considerably reduced for the well-behaving integral kernels, i.e., kernels reaching their extremes at the computational point and decreasing uniformly to zero. Figures 1 and 2 show that the kernels of the integrals 20 and 22 decrease to zero after a geocentric angle of about $15^{\circ}$. Therefore, by considering the inversion area larger by $15^{\circ}$ from each side, performing the inversion and selecting the result in the central area (smaller by $15^{\circ}$ ), the effect of spatial truncation error is reduced. Figures $4 \mathrm{a}$ and $4 \mathrm{~b}$ present the maps of the recovered SF by both integral formulae 20 and 22 , respectively, without removing the truncation error. As the figures show, the truncation error is considerably larger than the magnitude of SF in the marginal area. One issue which is visible in these figures is that the SF is 
smoother when it is recovered from $\delta T$ via the integral 20 as compared to the recovery by the integral 22 .

\subsubsection{Recovery of the stress function}

Now, the SST data are computed on the real orbit of the twin-satellites. The integrals 20 and 22 are used to invert them to the SF. In order to reduce the effect of the spatial truncation error, we selected the recovered SF in the central part which is smaller by $10^{\circ}$. Figures $5 \mathrm{a}$ and $5 \mathrm{~b}$ show the recovered SF by inverting the integrals 20 and 22 . The coastal lines and the tectonic boundaries have been shown in the map by black and green lines. The values of SF are larger when they are recovered from $\delta T$, whilst smaller from e $\delta \nabla T$. The maps clearly show that the SF is smoother in the former case than the latter. This could be expected as recovering high frequencies of the Earth gravity field from the potential observables is not possible, while first and second order derivatives of the potential are more capable for high resolution gravity field modelling from space.
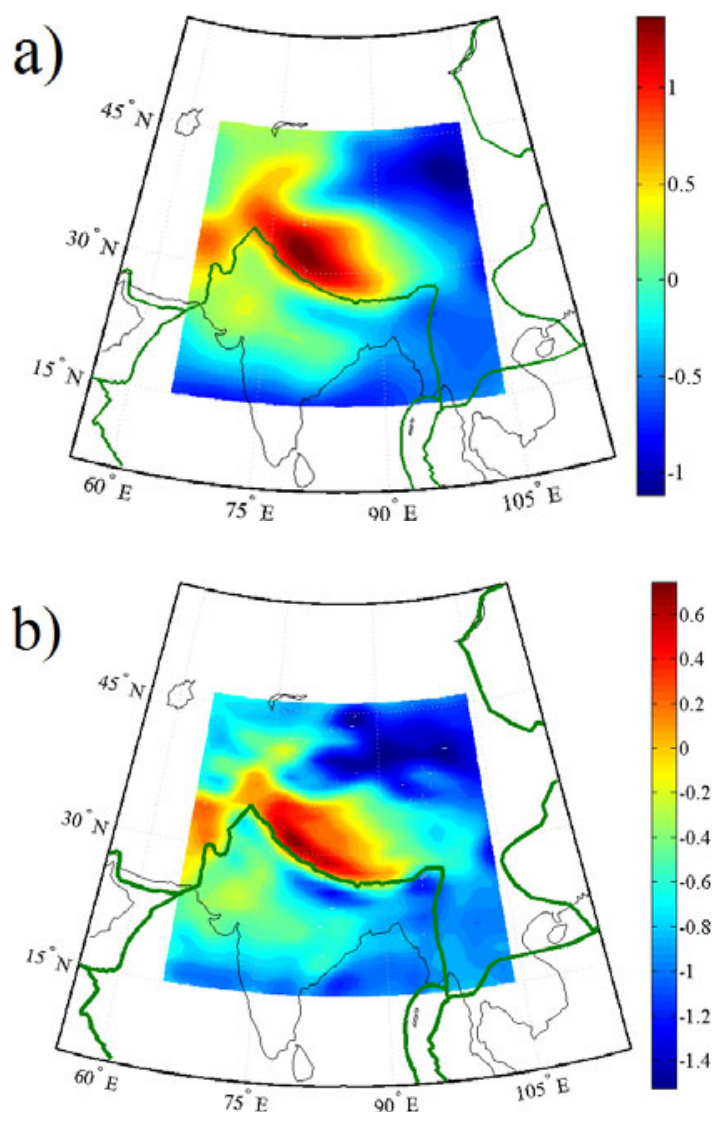

Fig. 5. SF recovered from: (a) disturbing potential differences $(\delta T)$, and (b) LOS gravity disturbances $(\mathbf{e} \cdot \delta \nabla T)$. Unit: $1 \mathrm{MPa}$. 


\subsubsection{Determination of sub-crustal stress from the recovered stress function}

Once SF is estimated, computation of its derivatives numerically towards the north and the east is straightforward. One can use the following formulae for generating the meridian and prime vertical sub-crustal stress components:

$$
S_{x}=-\frac{\Delta S}{\Delta \varphi}, \quad S_{y}=\frac{1}{\cos \varphi} \frac{\Delta S}{\Delta \lambda},
$$

where $S_{x}$ is the northward and $S_{y}$ is the eastward component, $\Delta S$ is the difference of the stress function between two adjacent cells towards the north or the east, $\Delta \varphi$ is the resolution in the northward direction and $\Delta \lambda$ in the eastward directions. Note that both of them should be in the radian units.

Here, we compute the magnitudes of the sub-crustal stress by:

$$
S_{T}=\sqrt{S_{x}^{2}+S_{y}^{2}},
$$
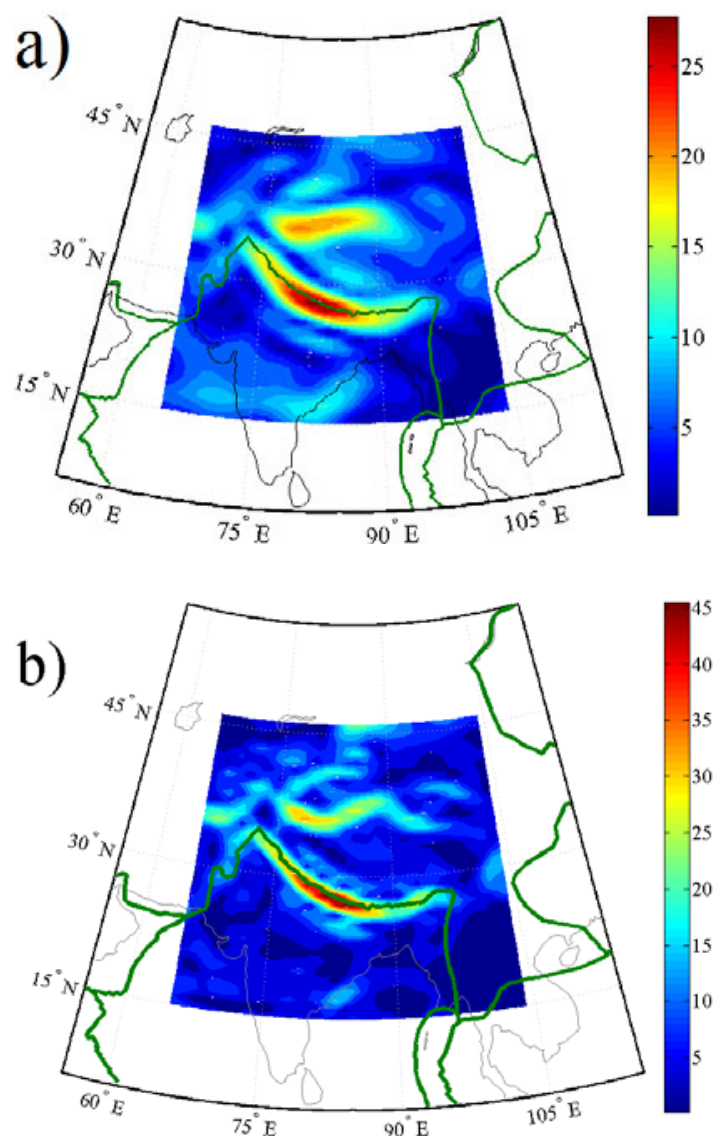

Fig. 6. Magnitudes of subcrustal stress derived from: (a) $\delta T$, and (b) $\mathbf{e} \cdot \delta \nabla T$. Unit: $1 \mathrm{MPa}$. 
and present them in the maps; see Fig. 6. Figure 6a shows the map of $S_{T}$ derived from the SF recovered from $\delta T$, and Fig. $6 \mathrm{~b}$ that from e $\delta \nabla T$. In spite of the fact that the values of SF presented in Fig. 5a are larger than those of Fig. 5b, the values of $S_{T}$ are smaller in Fig. 6a when they are derived numerically from the SF of Fig. 5a. The reason is that when sub-crustal stress components are computed from $\mathrm{SF}, \Delta S$ will be smaller in the case of using the SF generated from $\delta T$ than $\mathbf{e} \cdot \delta \nabla T$, which is due to the smooth nature of SF in the first case. Both maps of $S_{T}$, presented in Figs. 6a and 6b, show large subcrustal stress under the tectonic boundary in the southern part of Himalayas.

Table 2 presents the statistics of the recovered SF and the sub-crustal stress components recovered numerically from SF. According to the table, $S_{x}$ ranges from -28.27 to $21.58 \mathrm{MPa}$ when it is derived from $\delta T$, while for e $\delta \nabla T$ the range is from -46.42 to $32.02 \mathrm{MPa}$. The corresponding extreme values for $S_{y}$ are -9.12 to $14.11 \mathrm{MPa}$ for the former and from -19.28 to $23.75 \mathrm{MPa}$ for the latter functional.

Table 2

Statistics of recovered SF and sub-crustal stress components $S_{x}$ and $S_{y}$ derived from $\delta T$ and $\mathbf{e} \cdot \delta \nabla T$

\begin{tabular}{|c|c|c|c|c|c|}
\hline SST data & Quantity & Minimum & Maximum & Mean & Std. dev. \\
\hline \multirow{3}{*}{$\delta T$} & $S$ & -1.12 & 1.42 & -0.10 & 0.55 \\
& $S_{x}$ & -28.27 & 21.58 & -1.09 & 6.98 \\
& $S_{y}$ & -9.12 & 14.11 & -1.18 & 3.55 \\
\hline \multirow{3}{*}{$\mathbf{e} \cdot \delta \nabla T$} & $S$ & -1.52 & 0.79 & -0.68 & 0.45 \\
& $S_{x}$ & -46.42 & 32.02 & -0.23 & 9.43 \\
& $S_{y}$ & -19.28 & 23.75 & -0.98 & 4.93 \\
\hline
\end{tabular}

Note: Unit: $1 \mathrm{MPa}$.

\section{DISCUSSION}

In this study, we have used the SST data for recovering the sub-crustal stress components. Generally, a GGFM can be used for modelling these components by the Runcorn's formula. However, Eshagh (2016) showed that Runcorn's formula is not convergent for high degrees. This means that the idea of working with high degree GGFM is not successful.

The mantle convection occurs inside the Earth and only by investigating the long wavelength structure of the Earth gravity field, we can model and investigate it; see, e.g., Eshagh (2015), Eshagh and Tenzer (2015), and Tenzer and Eshagh (2015). The proper selection of the maximum degree of expansion and resolution of recovery play important roles in stress model- 
ling. Eshagh (2016) presented a formula for estimating the maximum degree of the expansion and the mean Moho depth. For example, for a mean Moho depth of $35 \mathrm{~km}$, the maximum expansion degree is 372 . The larger the Moho depth is the lower the maximum expansion degree will be.

Exploiting terrestrial gravity data means considering all frequencies of the gravity field implicitly. Therefore, the divergence occurs in the computations. In such a case, either kernel functions should be limited to a specific maximum degree or the integral inversion should be performed locally, while the local diverting frequencies of the system of equations are removed, e.g., using the truncated singular value decomposition technique; see Eshagh (2016).

The satellite data are more useful for stress modelling as the satellite altitudes filter out the high frequencies of the gravity field and represent the low frequencies better. For near sub-surface geophysical phenomena the lower altitude satellites are more suitable. On the other hand, for studying deeper structures of the Earth satellites flying at higher altitudes should be preferred. Consequently, SST data provided by GRACE should be more suitable for lithospheric stress modelling. Here, we have investigated two types of the SST data and concluded that $\mathbf{e} \cdot \delta \nabla T$ contains more frequencies of the gravity field and can present the stress better.

Eshagh (2015) reported that the sub-crustal stress reaches up to $100 \mathrm{MPa}$ over Himalayas, when a GGFM is considered to degree 360 with mean Moho depth of $23 \mathrm{~km}$. The magnitude of sub-crustal stress reaches to $5 \mathrm{MPa}$ in Taiwan (Tenzer and Eshagh 2015) using a GGFM and Eshagh and Tenzer (2015) reported a maximum of $70 \mathrm{MPa}$ when a mean Moho depth of $30 \mathrm{~km}$ is assumed. Eshagh (2016) reported maximum stress of about $60 \mathrm{MPa}$ over Iran. Eshagh and Romeshkani (2015) used the real GOCE data to recover the stress at the depth of $100 \mathrm{~km}$ and found that the stress reaches to about $100 \mathrm{MPa}$. The values that we have obtained for the stress based on our approaches are about 30 and $45 \mathrm{MPa}$ dependent on the use of $\delta T$ and $\mathbf{e} \cdot \delta \nabla T$ which are consistent with what can be obtained from other data types.

We emphasise that both of our methods have been applied under the same conditions, e.g., the same satellite orbits, spatial resolution, and the mean Moho depth. The difference between these two solutions is dependent on the type of observables and the mathematical models connecting the observables to the unknown SF. It is clear that the second method, which uses the LOS gravity disturbances, is capable of recovering higher frequencies of the sub-crustal stress as compared to disturbing potential differences. Consequently, we can conclude that exploiting the LOS gravity disturbances performs well and may be more suited for real applications. To prove this 
finding, recovery of sub-crustal stress from SST data contaminated by noise have to be performed. This task is left for our future research.

\section{CONCLUSIONS}

In this article two integral transformations between the stress function, numerical differentiation of which provides components of the sub-crustal stress, and the SST data have been derived. The first integral transformation assumes that the SST data are in the form of disturbing potential differences. SST data in the form of LOS gravity disturbance are related to the stress function in the second integral transformation.

The corresponding integral kernels have been investigated in the spatial domain showing their azimuthal dependence. The integral kernels are wellbehaving, thus suitable for solving the inverse problem, i.e., estimation of the stress function from the SST data. Direct and inverse problems have been tested in the numerical experiments. The results suggest that the stress function (and thus the components of the sub-crustal stress) estimated from the disturbing potential differences is smoother as compared to the estimate from the LOS gravity disturbances. This is due to the type of data being inverted and by using LOS gravity disturbances higher frequencies of the stress field can be recovered. Inversion of such data is better as long as the divergence does not happen in the mathematical model of the stress function. The theoretical apparatus may be applied for the recovery of the sub-crustal stress from satellite mission GRACE and its planned successor.

Acknowledgements. Michal Šprlák was supported by the project No. GA15-08045S of the Czech Science Foundation. Comments of the three anonymous reviewers are gratefully acknowledged.

\section{References}

Arabelos, D.N., and D. Tsoulis (2013), The exploitation of state of the art digital terrain databases and combined or satellite-only Earth gravity models for the estimation of the crust-mantle interface over oceanic regions, Geophys. $J$. Int. 193, 3, 1343-1352, DOI: 10.1093/gji/ggt081.

Block, A.E., R.E. Bell, and M. Studinger (2009), Antarctic crustal thickness from satellite gravity: Implications for the Transantarctic and Gamburtsev Subglacial Mountains, Earth Planet. Sci. Lett. 288, 1-2, 194-203, DOI: 10.1016/j.epsl.2009.09.022.

Bouman, J. (1998), Quality of regularization methods, DEOS Report no. 98.2, Delft University Press, Delft, The Netherlands. 
Braitenberg, C., and J. Ebbing (2009a), New insights into the basement structure of the West Siberian Basin from forward and inverse modeling of GRACE satellite gravity data, J. Geophys. Res. Solid Earth 114, B06402, DOI: 10.1029/2008JB005799.

Braitenberg, C., and J. Ebbing (2009b), The GRACE-satellite gravity and geoid fields in analysing large-scale, cratonic or intracratonic basins, Geophys. Prospect. 57, 4, 559-571, DOI: 10.1111/j.1365-2478.2009.00793.x.

Case, K., G. Kruizinga, and S.-C. Wu (2010), GRACE level 1B data product user handbook, Version 1.3, JPL D22027, Jet Propulsion Laboratory, California Institute of Technology, Pasadena, USA.

Chen, J.L., C.R. Wilson, B.D. Tapley, and S. Grand (2007), GRACE detects coseismic and postseismic deformation from the Sumatra-Andaman earthquake, Geophys. Res. Lett. 34, 13, L13302, DOI: 10.1029/2007GL030356.

Choi, S., C.W. Oh, and H. Luehr (2006), Tectonic relation between northeastern China and the Korean peninsula revealed by interpretation of GRACE satellite gravity data, Gondwana Res. 9, 1-2, 62-67, DOI: 10.1016/j.gr.2005. 06.002 .

Dai, C., C.K. Shum, R. Wang, L. Wang, J. Guo, K. Shang, and B. Tapley (2014), Improved constraints on seismic source parameters of the 2011 Tohoku earthquake from GRACE gravity and gravity gradient changes, Geophys. Res. Lett. 41, 6, 1929-1936, DOI:10.1002/2013GL059178.

ESA (1999), Gravity field and steady-state ocean circulation mission, Report for mission selection of the four candidate earth explorer missions, ESA SP1233(1), European Space Agency, ESA Publications Division, Noordwijk, The Netherlands.

Eshagh, M. (2011a), Sequential Tikhonov regularization: an alternative way for inverting satellite gradiometric data, $Z$. Vermessungs. 136, 2, 113-121.

Eshagh, M. (2011b), The effect of spatial truncation error on integral inversion of satellite gravity gradiometry data, Adv. Space Res. 47, 7, 1238-1247, DOI: 10.1016/j.asr.2010.11.035.

Eshagh, M. (2014), From satellite gradiometry data to sub-crustal stress due to mantle convection, Pure Appl. Geophys. 171, 9, 2391-2406, DOI: 10.1007/ s00024-014-0847-2.

Eshagh, M. (2015), On the relation between Moho and sub-crustal stress due to mantle convection, J. Geophys. Eng. 12, 1, 1-11, DOI: 10.1088/17422132/12/1/1.

Eshagh, M. (2016), Integral approaches to determine sub-crustal stress from terrestrial gravimetric data, Pure Appl. Geophys. 173, 3, 805-825, DOI: 10.1007/ s00024-015-1107-9.

Eshagh, M., and M. Romeshkani (2015), Determination of sub-lithospheric stress due to mantle convection using GOCE gradiometric data over Iran, $J$ Appl. Geophys. 122, 11-17, DOI: 10.1016/j.jappgeo.2015.08.001. 
Eshagh, M., and R. Tenzer (2015), Sub-crustal stress determined using gravity and crust structure models, Computat. Geosci. 19, 1, 115-125, DOI: 10.1007/ s10596-014-9460-9.

Fischell, R.E., and V.L. Pisacane (1978), A drag-free Lo-Lo satellite system for improved gravity field measurements. In: I. Mueller (ed.), Proc. Ninth Geodesy/Solid Earth and Ocean Physics (GEOP) Int. Symp. "Applications of Geodesy to Geodynamics”, 2-5 October 1978, Columbus Ohio State University, USA, Report No. 280, 213-219.

Fu, R.S. (1986), A numerical study of the effects of boundary conditions on mantle convection models constrained to fit the low degree geoid coefficients, Phys. Earth Planet. Int. 44, 3, 257-263, DOI: 10.1016/0031-9201(86) 90074-9.

Fu, R.S. (1990), The Earth's geoid anomalies and the physical mathematical model of the mantle convection, Chinese J. Geophys. 33 (Suppl. II), 457-468 (in Chinese).

Hajela, D.P. (1974), Improved procedures for the recovery of $5^{\circ}$ mean gravity anomalies from ATS-6/GEOS-3 satellite-to-satellite range-rate observations, Report No. 276, Department of Geodetic Science, Ohio State University, Columbus, USA.

Han, S.-C., J. Sauber, S.B. Luthcke, C. Ji, and F.F. Pollitz (2008), Implications of postseismic gravity change following the great 2004 Sumatra-Andaman earthquake from the regional harmonic analysis of GRACE intersatellite tracking data, J. Geophys. Res. 113, B11, B11413, DOI:10.1029/ 2008JB005705.

Hansen, P.C. (1998), Rank-deficient and Discrete Ill-posed Problems: Numerical Aspects of Linear Inversion, SIAM, Philadelphia, 243 pp.

Hansen, P.C. (2007), Regularization tools, version 4.0 for Matlab 7.3, Numer. Algorithms 46, 2, 189-194, DOI: 10.1007/s11075-007-9136-9.

Hofmann-Wellenhof, B., and H. Moritz (2005), Physical Geodesy, 2nd ed., Springer, Wien, 403 pp.

Huang, P.H., and R.S. Fu (1982), The mantle convection pattern and force source mechanism of recent tectonic movement in China, Phys. Earth Planet. Int. 28, 3, 261-268, DOI: 10.1016/0031-9201(82)90007-3.

Jekeli, C. (1999), The determination of gravitational potential differences from satellite-to-satellite tracking, Celest. Mech. Dyn. Astron. 75, 2, 85-101, DOI: 10.1023/A:1008313405488.

Kaban, M.K., and V. Trubitsyn (2012), Density structure of the mantle transition zone and the dynamic geoid, J. Geodyn. 59-60, 183-192, DOI: 10.1016/ j.jog.2012.02.007.

Kaban, M.K., M. Tesauro, and S. Cloetingh (2010), An integrated gravity model for Europe's crust and upper mantle, Earth Planet. Sci. Lett. 296, 3-4, 195-209, DOI: $10.1016 /$ j.epsl.2010.04.041. 
Keller, W., and M.A. Sharifi (2005), Satellite gradiometry using a satellite pair, J. Geodesy 78, 9, 544-557, DOI: 10.1007/s00190-004-0426-x.

Kiamehr, R., and L.E. Sjöberg (2006), Impact of a precise geoid model in studying tectonic structures - A case study in Iran, J. Geodyn. 42, 1-3, 1-11, DOI: 10.1016/j.jog.2006.04.001.

Kiamehr, R., M. Eshagh, and L.E. Sjöberg (2008), Interpretation of general geophysical patterns in Iran based on GRACE gradient component analysis, Acta Geophys. 56, 2, 440-454, DOI: 10.2478/s11600-007-0050-2.

Köther, N., H.-J. Götze, B.D. Gutknecht, T. Jahr, G. Jentzsch, O.H. Lücke, R. Mahatsente, R. Sharma, and S. Zeumann (2012), The seismically active Andean and Central American margins: Can satellite gravity map lithospheric structures? J. Geodyn. 59-60, 207-218, DOI: 10.1016/j.jog.2011.11.004.

Li, J., J. Chen, and Z. Zhang (2014), Seismologic applications of GRACE timevariable gravity measurements, Earthq. Sci. 27, 2, 229-245, DOI: 10.1007/ s11589-014-0072-1.

Liu, H.S. (1977), Convection pattern and stress system under the African plate, Phys. Earth Planet. Int. 15, 1, 60-68, DOI: 10.1016/0031-9201(77)90010-3.

Liu, H.S. (1978), Mantle convection pattern and subcrustal stress under Asia, Phys. Earth Planet. Int. 16, 3, 247-256, DOI: 10.1016/0031-9201(78)90018-3.

Liu, H.S. (1980a), Mantle convection and subcrustal stress under Australia, Mod. Geol. 7, 1, 29-36.

Liu, H.S. (1980b), Mantle convection and subcrustal stress under United States, Mod. Geol. 7, 81-93.

Liu, H.S., E.S. Chang, and G.H. Wyatt (1976), Small-scale mantle convection system and stress field under Pacific plate, Phys. Earth Planet. Int. 13, 3, 212217, DOI: 10.1016/0031-9201(76)90095-9.

Matsuo, K., and K. Heki (2011), Coseismic gravity changes of the 2011 Tohoku-Oki earthquake from satellite gravimetry, Geophys. Res. Lett. 38, 7, L00G12, DOI:10.1029/2011GL049018.

McAdoo, D.C., S.L. Farrell, S.W. Laxon, H.J. Zwally, D. Yi, and A.L. Ridout (2008), Arctic Ocean gravity field derived from ICESat and ERS-2 altimetry: Tectonic implications, J. Geophys. Res. 113, B5, B05408, DOI:10.1029/2007JB005217.

McKenzie, D.P. (1967), Some remarks on heat flow and gravity anomalies, J. Geophys. Res. 72, 24, 6261-6273, DOI:10.1029/JZ072i024p06261.

McNutt, M. (1980), Implication of regional gravity for state of stress in the Earth's crust and upper mantle, $J$ Geophys. Res. 85, B11, 6377-6396, DOI:10.1029/JB085iB11p06377.

Mikhailov, V., S. Tikhotsky, M. Diament, I. Panet, and V. Ballu (2004), Can tectonic processes be recovered from new gravity satellite data?, Earth Planet. Sci. Lett. 228, 3-4, 281-297, DOI: 10.1016/j.eps1.2004.09.035. 
Mikhailov, V., V. Lyakhovsky, I. Panet, Y. van Dinther, M. Diament, T. Gerya, O. deViron, and E. Timoshkina (2013), Numerical modelling of postseismic rupture propagation after the Sumatra 26.12.2004 earthquake constrained by GRACE gravity data, Geophys. J. Int. 194, 1, 640-650, DOI:10.1093/gji/ggt145.

Moritz, H. (2000), Geodetic reference system 1980, J. Geodesy 74, 1, 128-133, DOI: 10.1007/s001900050278.

Panet, I., F. Pollitz, V. Mikhailov, M. Diament, P. Banerjee, and K. Grijalva (2010), Upper mantle rheology from GRACE and GPS postseismic deformation after the 2004 Sumatra-Andaman earthquake, Geochem. Geophys. Geosyst. 11, 6, Q06008, DOI: 10.1029/2009GC002905.

Pavlis, N.K., S.A. Holmes, S.C. Kenyon, and J.K. Factor (2012), The development and evaluation of the Earth Gravitational Model 2008 (EGM2008), J. Geophys. Res. 117, B4, B04406, DOI: 10.1029/2011JB008916.

Pick, M. (1994), The geoid and tectonic forces. In: P. Vaníček and N.T. Christou (eds.), Geoid and its Geophysical Interpretations, CRC Press, Boca Raton, 239-253.

Pick, M., and I. Charvátová-Jakubcová (1988), Modification of the Runcorn's equations on the convection flows, Stud. Geophys. Geod. 32, 1, 47-53, DOI: 10.1007/BF01629000.

Pick, M., J. Pícha, and V. Vyskočil (1973), Theory of the Earth's Gravity Field, Elsevier, Amsterdam, 538 pp.

Reigber, C. (1989), Gravity field recovery from satellite tracking data. In: F. Sansó and R. Rummel (eds.), Theory of Satellite Geodesy and Gravity Field Determination, Lecture Notes in Earth Sciences, Vol. 25, Springer, Berlin Heidelberg, 197-234.

Reigber, C., H. Luehr, and P. Schwintzer (2002), CHAMP mission status, Adv. Space Res. 30, 2, 129-134, DOI: 10.1016/S0273-1177(02)00276-4.

Ricard, Y., L. Fleitout, and C. Froidevaux (1984), Geoid heights and lithospheric stresses for a dynamic Earth, Ann. Geophys. 2, 3, 267-286.

Ricard, Y., F. Chambat, and C. Lithgow-Bertelloni (2006), Gravity observations and 3D structure of the Earth, C. R. Geosci. 338, 14-15, 992-1001, DOI: 10.1016/j.crte.2006.05.013.

Rummel, R. (1980), Geoid heights, geoid height differences, and mean gravity anomalies from 'low-low' satellite-to-satellite tracking - an error analysis, Report No. 306, Department of Geodetic Science, Ohio State University, Columbus, USA.

Runcorn, S.K. (1964), Satellite gravity measurements and laminar viscous flow model of the Earth mantle, J. Geophys. Res. 69, 20, 4389-4394, DOI:10.1029/JZ069i020p04389. 
Runcorn, S.K. (1967), Flow in the mantle inferred from the low degree harmonics of the geopotential, Geophys. J. Int. 14, 1-4, 375-384, DOI: 10.1111/j.1365246X.1967.tb06253.x.

Seeber, G. (2003), Satellite Geodesy, 2nd ed., Walter de Gruyter, Berlin, 589 pp.

Shin, Y.H., H. Xu, C. Braitenberg, J. Fang, and Y. Wang (2007), Moho undulations beneath Tibet from GRACE-integrated gravity data, Geophys. J. Int. 170, 3, 971-985, DOI: 10.1111/j.1365-246X.2007.03457.x.

Souriau, M., and A. Souriau (1983), Global tectonics and the geoid, Phys. Earth Planet. Int. 33, 2, 126-136, DOI: 10.1016/0031-9201(83)90145-0.

Šprlák, M., and P. Novák (2014a), Integral transformations of deflections of the vertical onto satellite-to-satellite tracking and gradiometric data, J. Geodesy 88, 7, 643-657, DOI: 10.1007/s00190-014-0711-2.

Šprlák, M., and P. Novák (2014b), Integral transformations of gradiometric data onto GRACE type of observable, J. Geodesy 88, 4, 377-390, DOI: 10.1007/s00190-013-0689-1.

Sun, W. (2014), Recent advances of computing coseismic deformations in theory and applications, Earthq. Sci. 27, 2, 217-227, DOI: 10.1007/s11589-0140077-9.

Sun, W., and S. Okubo (2004), Coseismic deformations detectable by satellite gravity missions: A case study of Alaska $(1964,2002)$ and Hokkaido (2003) earthquakes in the spectral domain, J. Geophys. Res. 109, B4, B04405, DOI:10.1029/2003JB002554.

Tapley, B.D., S. Bettadpur, M. Watkins, and C. Reigber (2004), The gravity recovery and climate experiment: mission overview and early results, Geophys. Res. Lett. 31, 9, L09607, DOI:10.1029/2004GL019920.

Tedla, G.E., M.V.D. Meijde, A.A. Nyblade, and F.D.V.D. Meer (2011), A crustal thickness map of Africa derived from a global gravity field model using Euler deconvolution, Geophys. J. Int. 187, 1, 1-9, DOI:10.1111/j.1365246X.2011.05140.x.

Tenzer, R., and M. Eshagh (2015), Subduction generated sub-crustal stress in Taiwan, Terr. Atm. Oceanic Sci. 26, 261-268, DOI: 10.3319/TAO.2014.12. 04.01(T).

Tenzer, R., M. Eshagh, and S. Jin (2015), Martian sub-crustal stress from gravity and topographic models, Earth Planet. Sci. Lett. 425, 84-92, DOI: 10.1016/j.eps1.2015.05.049.

Tikhonov, A.N. (1963), Solution of incorrectly formulated problems and regularization method, Soviet Math. Dokl. 5, 1035-1038.

Tondi, R., R. Schivardi, I. Molinari, and A. Morelli (2012), Upper mantle structure below the European continent: Constraints from surface-wave tomography and GRACE satellite gravity data, J. Geophys. Res. 117, B9, B09401, DOI:10.1029/2012JB009149. 
von Frese, R.R.B., L.V. Potts, S.B. Wells, T.E. Leftwich, H.R. Kim, J.W. Kim, A.V. Golynsky, O. Hernandez, and L.R. Gaya-Piqué (2009), GRACE gravity evidence for an impact basin in Wilkes Land, Antarctica, Geochem. Geophys. Geosyst. 10, 2, Q02014, DOI:10.1029/2008GC002149.

Wang, L., C.K. Shum, F.J. Simons, B. Tapley, and C. Dai (2012), Coseismic and postseismic deformation of the 2011 Tohoku-Oki earthquake constrained by GRACE gravimetry, Geophys. Res. Lett. 39, 7, L07301, DOI:10.1029/ 2012 GL051104.

Wolff, M. (1969), Direct measurements of the Earth's gravitational potential using a satellite pair, J. Geophys. Res. 74, 22, 5295-5300, DOI:10.1029/ JB074i022p05295.

Zhao, S. (2013), Lithosphere thickness and mantle viscosity estimated from joint inversion of GPS and GRACE-derived radial deformation and gravity rates in North America, Geophys. J. Int. 194, 3, 1455-1472, DOI: 10.1093/gji/ ggt212.

Zuber, M.T., D.E. Smith, M.M. Watkins, S.W. Asmar, A.S. Konopliv, F.G. Lemoine, H.J. Melosh, G.A. Neumann, R.J. Phillips, S.C. Solomon, M.A. Wieczorek, J.G. Williams, S.J. Goosens, G. Kruizinga, E. Mazarico, R.S. Park, and D.-N. Yuan (2013), Gravity field of the Moon from the gravity recovery and interior laboratory (GRAIL) mission, Science 339, 6120, 668-671, DOI: $10.1126 /$ science. 1231507 .

Received 21 November 2014 Received in revised form 22 July 2015

Accepted 26 August 2015 